\title{
Freiräume als Orte der Begegnung
}

\section{Planerische Leitbilder und alltägliche Erfahrungen in superdiversen Stadtquartieren}

\author{
Charlotte Räuchle ${ }^{1}$ Ulrich Berding ${ }^{2}$ \\ Online publiziert: 19. Mai 2020 \\ (c) Der/die Autor(en) 2020
}

\section{Zusammenfassung}

Die Relevanz von Begegnungen in öffentlichen Räumen für das gesellschaftliche Zusammenleben in urbanen Quartieren und die Bedeutung der Gestaltung des Stadtraums sind viel diskutierte Themen in Stadtforschung und -planung. Meist werden die damit verbundenen Chancen, über solche Begegnungen die Akzeptanz gesellschaftlicher Vielfalt zu steigern, und die damit verbundenen Herausforderungen, die öffentliche Räume als Orte gesellschaftlicher Konflikte mit sich bringen können, kritisch gegenübergestellt und gegeneinander abgewogen. Obwohl also ein breites Wissen über öffentliche Räume als Orte der Begegnung besteht, ist es dennoch notwendig, seine Gültigkeit immer wieder in Bezug auf bestimmte lokale Kontexte zu überprüfen. Am Beispiel des superdiversen hannoverschen Quartiers Sahlkamp-Mitte kontrastiert der Artikel die der Freiraumgestaltung zugrunde liegenden planerischen Ideen mit alltäglichen Erfahrungen vom Zusammenleben der Bewohnerinnen und Bewohner. Es zeigt sich, dass hier Diskrepanzen bestehen und auch „organisierte Begegnungen“ im Freiraum einen produktiven Beitrag zum ,guten Zusammenleben“ im Quartier leisten können.

Schlüsselwörter Nachbarschaft · Interaktionen · Öffentlicher Raum · Sozialer Zusammenhalt · Stadtplanung

\section{Public spaces as sites of encounter}

Planning ideas and everyday experiences in superdiverse urban neighbourhoods

\begin{abstract}
The relevance of encounters in public spaces for the social cohesion in urban neighbourhoods and the role of the urban design in this context are highly discussed topics in urban research and planning. On the one hand, encounters in public spaces are said to increase the acceptance of social diversity but on the other hand research stresses that open spaces can become places of social conflict. Thus, even if there is a broad knowledge of public spaces as places of encounter, it is still necessary to continually test its validity with respect to specific local contexts. Taking the superdiverse Hanoverian neighbourhood Sahlkamp-Mitte as an example, this article contrasts the planning ideas underlying the open space development with everyday experiences of neighbourhood residents. The article demonstrates the inherent discrepancies and shows that organized encounters in public spaces can also foster a communal life characterized by dialogue and neighbourly solidarity.
\end{abstract}

Keywords Social cohesion · Interactions $\cdot$ Community $\cdot$ Social conflict $\cdot$ Urban planning

Charlotte Räuchle

charlotte.raeuchle@fu-berlin.de

Ulrich Berding

berding@plan-zwei.com
1 Institut für Geographische Wissenschaften, Freie Universität Berlin, Malteserstr. 74-100, 12249 Berlin, Deutschland

2 plan zwei, Postkamp 14 a, 30159 Hannover, Deutschland 


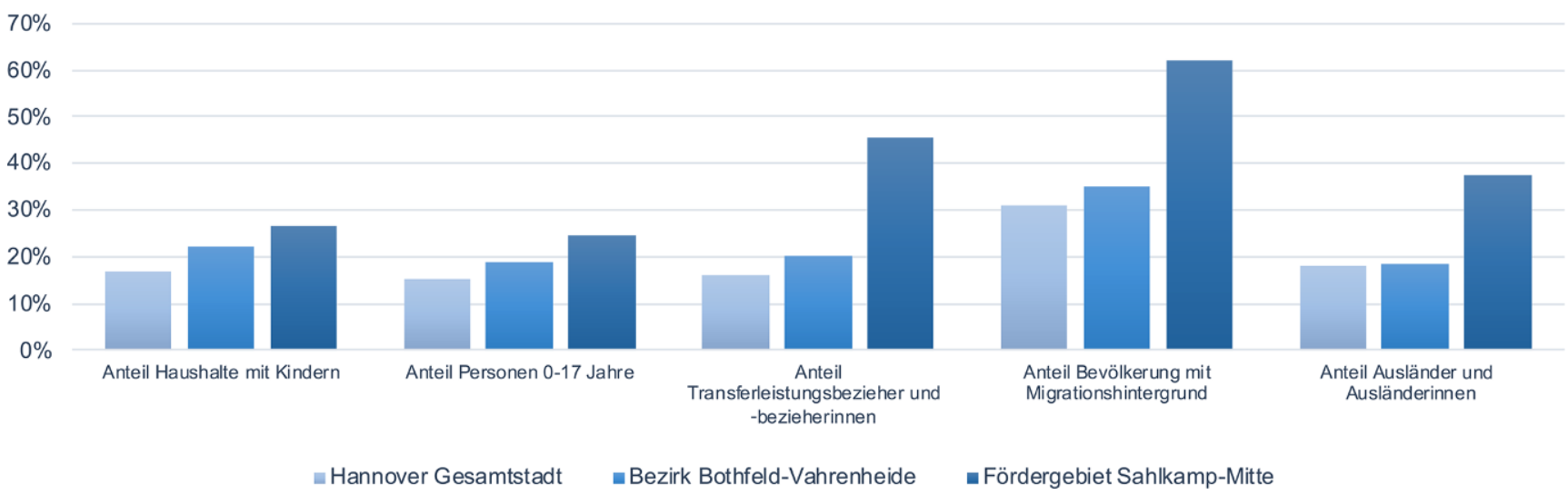

Abb. 1 Soziodemografische Daten zu Sahlkamp-Mitte im Vergleich zur Gesamtstadt Hannover und dem Bezirk Bothfeld-Varenheide, Stichtag 31.12.2017. (Quelle: eigene Darstellung nach LHH 2018 sowie Landeshauptstadt Hannover, Fachbereich Personal und Organisation, Sachgebiet Wahlen und Statistik)

Die Bedeutung von Begegnungen in öffentlichen Räumen für das gemeinschaftliche Zusammenleben in urbanen Quartieren und der Einfluss des physisch-materiellen Raums hierbei sind viel diskutierte Themen in der angewandten Geografie sowie der interdisziplinären Stadtforschung und -planung. Dabei werden die damit verbundenen Chancen - über solche Begegnungen soll die Akzeptanz gesellschaftlicher Vielfalt gesteigert werden - und die damit verbundenen Herausforderungen - öffentliche Räume können Orte gesellschaftlicher Konflikte sein - meist kritisch gegenübergestellt. Obwohl es also ein breites Wissen über öffentliche Räume als Orte der Begegnung gibt, muss dennoch seine Gültigkeit immer wieder in Bezug auf bestimmte lokale Kontexte überprüft werden - auch deshalb, weil Stadtgesellschaften einem stetigen, u. a. migrationsbedingten Wandel unterworfen sind und Nachbarschaften in der Struktur ihrer Anwohnerinnen und Anwohner vielfältiger werden (Vertovec 2007). Diese Beobachtung trifft auf unterschiedliche lokale Settings und Quartierstypen $\mathrm{zu}$, wobei immer auch die städtebaulich-planerische Dimension eine Rolle bei öffentlichen Räumen als Orten der Begegnung spielt.

Am Beispiel des superdiversen hannoverschen Quartiers Sahlkamp-Mitte zeigt der Artikel auf, wie „erfolgreich“ öffentlich zugängliche Freiräume als Begegnungsorte sind. Kontrastiert werden dabei die der Freiraumgestaltung zugrunde liegenden planerischen Ideen mit den alltäglichen Erfahrungen der Anwohnerinnen und Anwohner. Außerdem werden quartiersbezogene Initiativen der sozialen Stadtentwicklung hinsichtlich der Frage vorgestellt, wie wichtig sie für die Organisation von Freiräumen als Treffpunkte sind.

Der Artikel basiert auf dem vom „Bundesministerium für Bildung und Forschung“ finanzierten Verbundprojekt „KoopLab: Teilhabe durch kooperative Freiraumentwicklung in Ankunftsquartieren“, das an den drei Standorten Dort- mund, Leipzig und Hannover seit 2018 angesiedelt ist und partizipative Formate der Freiraumnutzung erprobt (www. kooplab.de). Der Standort Hannover wird durch das „Institut für Geographische Wissenschaften“" an der Freien Universität Berlin, das hannoversche Büro „plan zwei“ sowie die „Internationalen StadtteilGärten Hannover e. V.“ (ISG) betreut. In diesem Rahmen wurde auch das empirische Material erhoben, auf dem dieser Artikel beruht.

Sahlkamp-Mitte mit seinen gut 5600 Einwohnerinnen und Einwohnern und knapp 2500 Haushalten liegt am nordöstlichen Rand der Landeshauptstadt Hannover und gehört zum Stadtbezirk Bothfeld-Vahrenheide. Das Quartier wurde seit den 1960er Jahren unter Federführung der öffentlichen Hand u.a. in Kooperation mit dem Wohnungsunternehmen „Neue Heimat“ errichtet, wobei der Fokus auf dem sozialen Wohnungsbau lag. Seit 2009 ist das Quartier als „Stadtteil mit besonderem Entwicklungsbedarf" Teil des BundLänder-Programms „Soziale Stadt“ (LHH 2015, S. 5) und wurde 2010 förmlich als Sanierungsgebiet ausgewiesen. Es weicht soziodemografisch zum Teil vom gesamtstädtischen und bezirksweiten Durchschnitt ab (s. Abb. 1). So ist es beispielsweise von einem überdurchschnittlich hohen Anteil an kinderreichen Haushalten, Kindern und Jugendlichen unter 18 Jahren, hohen Werten in puncto Transferleistungsbezieherinnen und -beziehern sowie an Deutschen mit Migrationshintergrund geprägt.

\section{Begegnungen in (grünen) Freiräumen in Stadtforschung und Planungstheorie}

Zunächst gilt, dass die Idee von der zentralen Stellung öffentlicher Räume, ihrer Gestaltung und Nutzung für das Leben in der Stadt, eine Binsenweisheit ist (Carmona et al. 2008; Jacobs 1993 (1963); Madanipour 2010). In den stadtund planungstheoretischen Debatten herrscht jedoch Un- 
einigkeit darüber, welche Bedeutung öffentlich nutzbare (grüne) Freiräume als Orte der Begegnung tatsächlich haben. Manche Autorinnen und Autoren sind davon überzeugt, dass öffentliche Räume als gemeinsam genutzte Alltagsorte den Kontakt und Austausch zwischen Angehörigen unterschiedlicher gesellschaftlicher Gruppen, also Begegnung mit gesellschaftlicher Differenz fördern (Sennett 1991). Dadurch, dass sie Bewegungsraum für unterschiedliche Menschen sind und man hier (absichtslos) auf anderes Verhalten, unterschiedliche Interessen und Sichtweisen treffen kann, ,findet hier der soziale Austausch statt, der erst eine soziale Mischung zum integrativen Erfolgsmodell macht oder aber zur Entfremdung und Feindseligkeit führt" (Dangschat 2011, S. 4). Öffentliche Räume können damit als Begegnungs- und Erfahrungsräume bedeutende Lernfelder für Toleranz und Akzeptanz darstellen: ,[T]hey are one of the few remaining loci where we can encounter difference and learn to understand and tolerate other people" (Shaftoe 2008, S. 5).

Skeptische Stimmen warnen jedoch vor der Zelebrierung und Romantisierung öffentlicher Räume als potenzielle Orte der Begegnung. Sie bezweifeln, dass öffentliche Räume selbst wenn sie „gut“" gestaltet sein mögen - tatsächlich Orte gleichberechtigter Teilhabe sind, da ,das alltägliche Interaktionsgeschehen nicht von Kontakt und Austausch, sondern von gegenseitiger Distanzierung und Gleichgültigkeit bestimmt ist" (Wiesemann 2015, S. 51). Viele Begegnungen in öffentlichen Räumen sind hiernach lediglich flüchtig und oberflächlich (Amin 2002). Der Beitrag öffentlicher Räume zur Intensivierung von sozialen Bezügen zwischen unterschiedlichen Menschen und Gruppen, um Ansichten und Haltungen gegenüber Differenz positiv zu verändern, sei daher gering (Valentine 2008). Eher sei es so, dass durch Begegnung mit Differenz in öffentlichen Räumen bestehende Vorurteile und Fremdbilder bekräftigt anstatt aufgebrochen würden. Öffentliche Räume werden hier als ,zones of encounter" (Wood und Landry 2008, S. 105) also als ein äußerst ambivalentes Terrain dargestellt.

Als eine Ursache für die unterschiedliche Bewertung der Möglichkeiten und Grenzen der sozialen Integrationsfunktion von Freiräumen ist die Tatsache zu sehen, dass die (potenziellen) Nutzerinnen und Nutzer selbst stark zu differenzieren sind und unterschiedliche Voraussetzungen für Kommunikation und Interaktion mitbringen. So empfinden unterschiedliche soziale Gruppen öffentliche Räume unterschiedlich attraktiv und haben zudem spezifische Nutzungsbedürfnisse und Vorlieben. Mit einer stetig vielfältiger werdenden Gesellschaft differenzieren sich auch die Nutzungsanforderungen an die öffentlichen Räume weiter aus. Darüber hinaus belegen sozialwissenschaftliche Untersuchungen, dass Angehörige sozialer Gruppen Nähe untereinander suchen und bestrebt sind, sich anderen Gruppen gegenüber abzugrenzen: „Tatsächlich steht einem nichts ferner und ist nichts weniger tolerierbar als Menschen, die sozial fern stehen, aber mit denen man in räumlichen Kontakt kommt" (Bourdieu 1991, S. 32).

Insgesamt wird die Frage, welchen Beitrag die planerische Gestaltung öffentlicher Räume zur Schaffung von Begegnungen leistet, unterschiedlich bewertet (vhw 2017). Einerseits stellt z.B. Gehl (1987) eindeutig einen Zusammenhang zwischen der Qualität des physischen Raums und den in ihm ausgeführten Aktivitäten fest. Andererseits wird die physische Gestalt des öffentlichen Raums als Begegnungsort als nachrangig eingeschätzt. Planung könne dabei durch bauliche Gestaltung lediglich Kontaktanlässe von sich fremden Personen schaffen (Dangschat 2011; Wiesemann 2015). Auch könnten planerische Nutzungsfestlegungen in Konkurrenz zur Idee von Freiräumen als Begegnungsorten treten. Das gilt beispielsweise für den ruhenden und fließenden Verkehr, der nicht nur Flächen in Anspruch nimmt, sondern schwer überwindbare Barrieren in öffentlichen Räumen schaffen kann. Die „gute“ Gestaltung des physischen Raums ist also eine Voraussetzung, aber nicht die entscheidende für Begegnungen. Jedoch gilt insgesamt: „Räume, die ausreichend groß, nutzungs(offen) bzw. divers gestaltet sind, so dass unterschiedlichste Nutzergruppen ihre Nische finden und sich nicht gegenseitig ausschließen, [...] fördern eine Anwesenheit verschiedener Menschen." (vhw 2017, S. 47) Hier kann eingewendet werden, dass planerische Leitbilder oft (Fehl-)Prognosen für zu bauende Quartiere entwickeln und die Auswertung der dortigen Erfahrungen nachträglich erfolgt.

Es ergeben sich also verschiedene Möglichkeiten für „Momente der Begegnung“ in öffentlichen Freiräumen (Wiesemann 2015). So können diese als Begegnungsorte bestehende Meinungen gegenüber anderen sozialen Gruppen festigen und vorhandene Vorurteile stärken. Ebenso können öffentliche Räume aber auch wichtige Kontakte ermöglichen. Wie stellt sich dies nun für Sahlkamp-Mitte dar?

\section{(Grüne) Freiräume in Sahlkamp-Mitte: geplante Begegnungen?}

Erst Ende der 1950er Jahre wurde das Quartier in die städtische Planung aufgenommen, da es aufgrund seiner Lage an der Stadtgrenze lange Zeit nicht von Interesse war (Bochynek-Friske 2001). Insgesamt waren die Ideen für seine Entwicklung typisch für den sozialen Wohnungsbau der 1960/70er Jahre. So liegt dem Quartier baustrukturell ein einheitliches Konzept des zeittypischen ,gegliederten und aufgelockerten" Städtebaus mit sich wiederholendem Bebauungsmuster in Fertigbauweise zugrunde (LHH 2015, S. 22). Es wird dominiert von 4- bis 12-geschossigen Wohnanlagen und großen Mehrfamilienhäusern, die als Woh- 


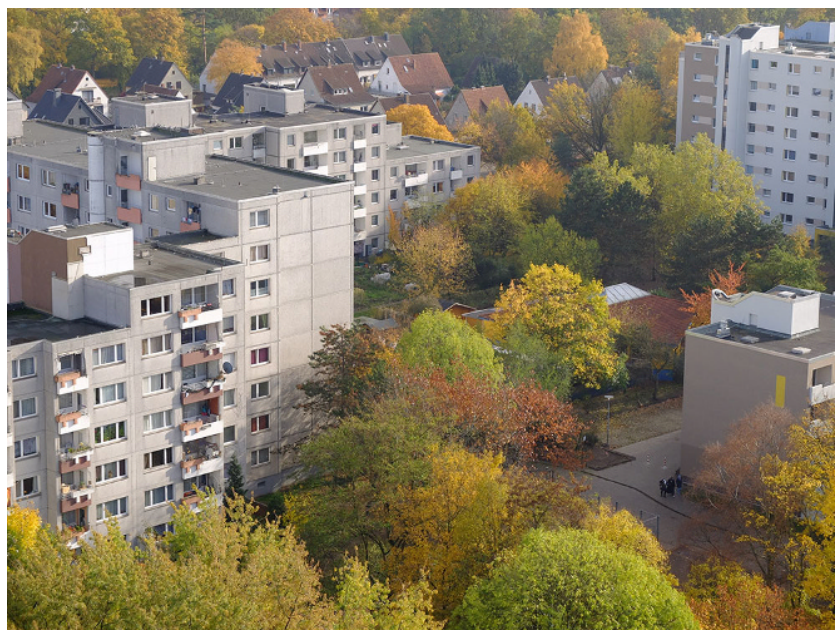

Abb. 2 Sahlkamp-Mitte von oben. (Quelle: plan zwei)

nungseigentümergemeinschaften (WEGs) organisiert oder im Besitz von Wohnunternehmen und Immobilieninvestoren sind. Daneben gibt es Einfamilien- und Reihenhäuser (EFH) (s. Abb. 2). Dieses Muster des Zeilenbaus mit prägenden Hochhäusern in der Mitte und niedrigen Gebäuden an den Rändern ist ebenfalls zeittypisch. Als Ausdruck des Leitbildes der autogerechten Stadt kennzeichnet zudem ein hoher Anteil an Verkehrsflächen das Gebiet.

Aus dieser städtebaulichen Struktur ergeben sich weitläufige, aber auch monotone und wenig geschützte Grünflächen und Freiräume zwischen den frei im Raum angeordneten Hochhäusern. Dadurch entstehen oft diffuse und wenig zusammenhängende Zwischenbereiche, die sich nur schwer privat nutzen lassen. In der ursprünglichen Konzeption des Quartiers waren zwar bestimmte Räume der Begegnung geplant, z.B. Sportplätze oder der SahlkampMarkt, spontane Aneignungen der grünen Freiräume und damit deren Nutzung als Treffpunkte waren jedoch nicht vorgesehen.

Im Kontext der Sozialen Stadt gewannen einige Jahrzehnte nach der baulichen Fertigstellung des Quartiers die Sanierung und die Instandsetzung der Freiräume an Bedeutung, wobei auch ihre Aufenthaltsqualität im Vergleich zu ihrem ursprünglichen Zustand verbessert werden sollte (LHH 2013). Die kommunalen Vertreterinnen und Vertreter erhofften sich von einer Neugestaltung der Außenräume auch eine „Befriedung“ des Quartiers und einen positiven Effekt auf das soziale Zusammenleben, so wie es in der Forschung vermutet wird. Für die Gestaltung von Begegnungsmöglichkeiten gibt es daher aktuelle Beispiele. Es ist nicht nur der sog. ,Stadtteilpark“ umfassend saniert worden, der seitdem u. a. von Kindern und Jugendlichen deutlich intensiver genutzt wird, auch eine (temporäre) BouleBahn zieht Anwohnerinnen und Anwohner an und schafft damit neue Möglichkeiten der Begegnung.

\section{Erfahrungen im Quartier: (grüne) Freiräume als alltägliche Begegnungsorte?}

Diese materiell-physische Gestaltung der Freiräume durch die Stadt- und Raumplanung stellt gewissermaßen die Rahmenbedingungen für Begegnungen dar. Wie aber erleben Anwohnerinnen und Anwohner in ihrem Alltag die öffentlichen Freiräume als Begegnungsorte? Viele unterscheiden häufig zwischen funktionalen und sozialen Aspekten (Gehl 1987): Während die Darstellung funktionaler Tätigkeiten überwiegt (,zum Einkaufen gehen“, „den Hund spazieren führen"), schildern einige, dass sie im öffentlichen Raum ihre Freizeit verbringen und Bekannte auf dem SahlkampMarkt, zum Grillen im Park oder beim Boule-Spielen treffen. Es käme jedoch wenig zu flüchtigen Begegnungen, geschweige denn zu engeren Kontakten mit vorher unbekannten Personen - was gewissermaßen die pessimistischen Annahmen zur geringen Relevanz von Begegnungen im öffentlichen Raum für das Zusammenleben im Quartier zu bestätigen scheint. Im Gegensatz dazu sind besonders Kinder und Jugendliche viel im Quartier unterwegs und treffen sich „draußen“, d.h. nutzen die Grün- bzw. Freiflächen aktiv und bewusst.

Es zeigt sich, dass Begegnungen, wenn, v. a. zwischen sich sozial nahestehenden Gruppen stattfinden. So kommen im Stadtteilpark z. B. die Bewohnerinnen und Bewohner der umliegenden Hochhäuser zusammen. Anwohnerinnen und Anwohner der WEGs und der EFHs halten sich hier dagegen nicht lange auf, während sich im Bereich der WEGs und der EFHs wiederum Anwohnerinnen und Anwohner aus dem Bereich des sozialen Wohnungsbaus selten treffen. Anders als vermutet, werden also nicht alle Freiräume in Sahlkamp-Mitte trotz ihrer (teilweise) offenen und unterschiedlichen Gestaltung von verschiedenen Anwohnerinnen- und Anwohnergruppen gleichermaßen in Anspruch genommen. Es gibt jedoch zentrale Orte, die verschiedene soziale Milieus nutzen und an denen es zu flüchtigen, situativen Begegnungen kommt - z.B. das Einkaufszentrum, die Stadtbahnhaltestellen oder der Freiraum vor sozialen Einrichtungen wie Kitas etc.

Wenn soziale Nähe entsteht, bedeutet dies jedoch nicht automatisch Konfliktfreiheit. Die Schilderungen des Verhaltens anderer Personen im öffentlichen Raum schwanken zwischen Akzeptanz und Ablehnung. Ablehnung erfolgt dann, wenn von bestimmten Normen abgewichen wird. Die Grenzen, entlang derer dies ausgehandelt wird, sind situativ und kontextbezogen zu bestimmen. Es werden gängige Verhaltensweisen kritisiert („Grillen im Park“, „laut spielende Kinder") und diese bestimmten Gruppen zugeschrieben. Bestehende Stereotype und Vorurteile werden also durchaus reproduziert. Der öffentliche Raum dient auch als Reflexionsfläche für die Wahrnehmung der Sicherheit im Sahlkamp. Einerseits wird immer wieder von einem Gefühl der 
Unsicherheit durch ganz unterschiedliche Gruppen von Bewohnerinnen und Bewohnern berichtet. Dem steht die Erfahrung anderer Anwohnerinnen und Anwohner entgegen, denen im Sahlkamp ,noch nie etwas Schlimmes“ passiert sei. Das medial verbreitete negative Image sei viel schlechter als das „wirkliche Leben“ im Quartier.

Die Verhandlung über die Nutzung des öffentlichen Raums wird damit zu einer Verhandlung über die Qualität des Zusammenlebens im Quartier. Deutlich wird, dass Begegnungen im Freiraum sehr unterschiedlich bewertet werden, durchaus abhängig von Geschlecht, Alter, Ethnizität etc. Zwar erwähnen Anwohnerinnen und Anwohner flüchtige Kontakte, doch die Grün- bzw. Freiflächen werden auch als geteilter Raum wahrgenommen, den nicht alle Anwohnerinnen und Anwohner überall zu jeder Zeit nutzen.

\section{Rolle der sozialen Quartiersentwicklung: "organisierte Begegnungen"?}

In der Wahrnehmung der Anwohnerinnen und Anwohner scheinen also Begegnungen und Kontakte im öffentlichen Raum weniger bedeutsam zu sein. Zwar hat die Stadtplanung hinsichtlich der materiell-physischen Gestaltung des Stadtraums verschiedene Schritte unternommen, um diesen für Kontakte und Begegnungen attraktiver zu machen. Diese Maßnahmen zeigen durchaus auch Erfolge. Zugleich lohnt ein Blick auf Projekte der organisierten Zivilgesellschaft, des Quartiersmanagements (QM) und der Gemeinwesenarbeit (GWA), die gezielt Anlässe für nachbarschaftliche Begegnungen in den öffentlichen Räumen des Quartiers schaffen und darüber einen positiven Beitrag zum Zusammenleben leisten wollen. Derartige Ansätze, die (interkulturelle) Begegnungen organisieren, sind u. a. deshalb kritisiert worden, weil sie Teil sozialräumlich ausgerichteter, staatlicher Integrationspolitiken seien (Lapina 2017). Die tatsächlichen Bedürfnisse der Anwohnerinnen und Anwohner würden dabei nur unzureichend berücksichtigt. Das ändert aber nichts daran, dass immer wieder auch auf den positiven Effekt dieser Räume für das nachbarschaftliche Zusammenleben verwiesen wird, z.B. bei Gemeinschaftsgärten (BBSR 2015).

Auch in Sahlkamp-Mitte gibt es solche Projekte, die Begegnungen und Kontakte im (halb)öffentlichen Raum fördern und dabei gezielt Treffpunkte schaffen wollen. Besonders bekannt sind nicht nur im Quartier, sondern im gesamten Stadtraum die 2007 gegründeten ISG. In Sahlkamp-Mitte betreiben sie zwei Gärten, die sich auf Tiefgaragendächern zwischen mehrgeschossigen Häusern befinden (s. Abb. 3). Da diese vorher bloße Abstandsflächen waren, fand hier eine explizite Umnutzung bzw. Umdeutung der Jahrzehnte vorher errichteten Freiräume statt. Diese Gär-

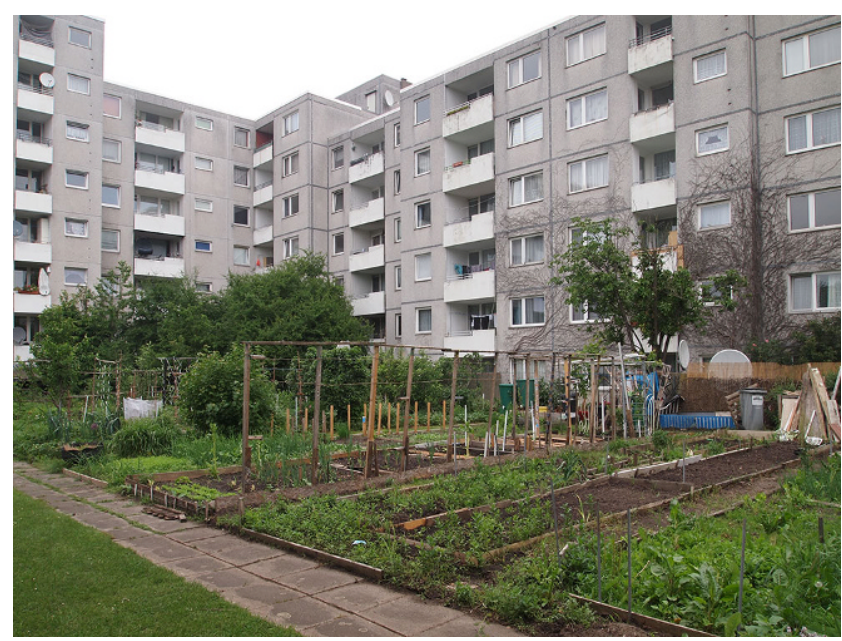

Abb. 3 Die ISG-Gärten auf Tiefgaragendächern in Sahlkamp-Mitte. (Quelle: plan zwei)

ten sind halböffentlich zugänglich. Die beteiligten Familien können Gemüse und Blumen anpflanzen. Die Gärten ,wollen ein Stück Heimat für MigrantInnen und Deutsche sein, wo sie sich geborgen fühlen, gemeinsam etwas ansäen, pflegen und ernten" (ISG Hannover 2017). Die ISG veranstalten zudem Feste, und auch dadurch werden die Gärten zu tatsächlichen Treffpunkten. Laut eigenen Angaben werden sie regelmäßig von 300 bis 400 Personen genutzt (BBSR 2015, S. 68). Besonders wichtig für die Arbeit im Quartier ist, dass die ISG mit vielen Gärtnerinnen und Gärtnern gut vernetzt sind und darüber verschiedene Anwohnerinnen- und Anwohnergruppen erreichen.

Während sich also die Stadtentwicklung im Kontext der Sozialen Stadt um eine Modernisierung vorhandener Freiräume und Grünflächen bemüht, nutzen die Stadtteilgärten brachliegendes Flächenpotenzial sichtbar um. Das Projekt KoopLab versucht dagegen, punktuell Begegnungsanlässe an verschiedenen Orten im öffentlichen Raum zu schaffen. Ein mobiler Bauwagen diente z. B. von Frühjahr bis Herbst 2019 als temporärer, nachbarschaftlicher Treffpunkt an wenig genutzten Freiräumen im Quartier (s. Abb. 4). An einem Standort kamen besonders viele Kinder, an einem anderen ältere, sich vor unbekannte Anwohnerinnen und Anwohner vorbei, um sich über ihr Leben im Quartier auszutauschen. Der Bauwagen funktionierte also durchaus als ein Beispiel dafür, wie Orte der „geselligen Begegnung“ (Wiesemann 2015) in Freiräumen geschaffen werden können, wenn auch seine Wirkung nicht überschätzt werden darf. Ob der hohe organisatorische und personelle Aufwand gerechtfertigt ist, sei dahingestellt. 
Abb. 4 Der KoopLab-Bauwagen in Sahlkamp-Mitte. (Quelle: plan zwei)

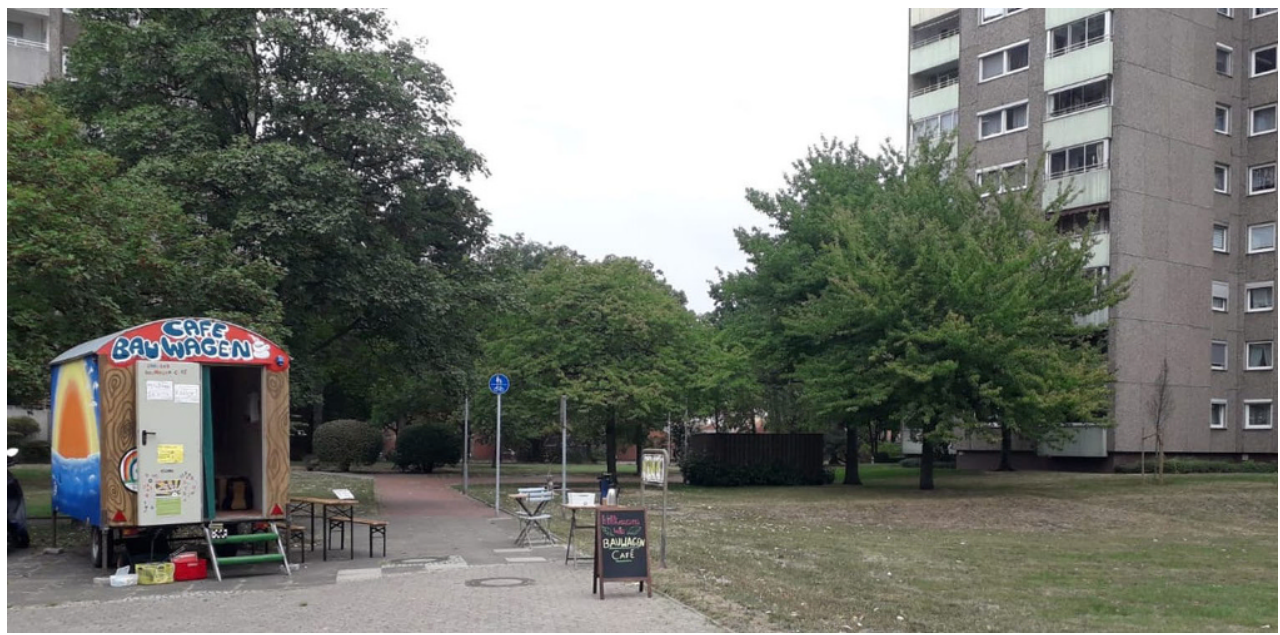

\section{Begegnungen im öffentlichen Raum superdiverser Stadtquartiere}

Die Bedeutung von Grün- und Freiflächen für spontane Begegnungen und Kontakte in urbanen Nachbarschaften wird von der Forschung einerseits fast euphorisch hervorgehoben, andererseits eher pessimistisch eingeschätzt. Dabei wird auch die Relevanz der planerischen Gestaltung des Freiraums unterschiedlich beurteilt. Das hannoversche Beispiel Sahlkamp-Mitte macht Folgendes deutlich: Während in der ursprünglichen Planung des Quartiers zunächst nur wenige Freiräume explizit als Treffpunkte vorgesehen waren, hat sich dieser ,planerische Blick“ gewandelt, und die Stadt bemüht sich durch investive Maßnahmen, nicht nur die Aufenthaltsqualität zu steigern, sondern damit auch das soziale Zusammenleben im Stadtteil zu verbessern - durchaus mit Erfolg. Die Erfahrungen der Anwohnerinnen und Anwohner hinsichtlich des öffentlich zugänglichen Freiraums als Begegnungsort sind nichtsdestotrotz zwiespältig: Es kommt, ihrer Wahrnehmung nach, wenig zu flüchtigen oder sogar engeren Begegnungen mit vorab fremden Personen und wenn, dann sind diese nicht zwangsläufig konfliktfrei. Der von der Planung zunächst für alle zugänglich gestaltete Freiraum wird also durchaus auch als geteilter Raum wahrgenommen. Dass Menschen positive Erfahrungen mit Begegnungen in öffentlichen Räumen machen, kann jedoch durch „organisierte Begegnungen“, die z.B. von den QMs oder der GWA initiiert werden, gefördert werden. Hannover-Sahlkamp wandelt sich in seiner Struktur zurzeit, u. a. aufgrund der anhaltenden Zuwanderungsbewegungen und eines immer enger werdenden Wohnungsmarkts. Während sich am Quartier also verdeutlichen lässt, dass man die Relevanz des Freiraums für Begegnungen derzeit weder über- noch unterschätzen darf, spricht viel dafür, dass seine Bedeutung für das soziale Zusammenleben vor Ort eher zunehmen wird.
Danksagung Wir bedanken uns beim Bundesministerium für Bildung und Forschung für die Förderung des Projekts „KoopLab: Teilhabe durch kooperative Freiraumentwicklung in Ankunftsquartieren“, auf dem dieser Beitrag beruht. Des Weiteren bedanken wir uns bei Maximilian Rohland für seine konstruktive Kritik sowie das Erstellen der Abbildungen. Schließlich geht unser Dank an zwei anonyme Reviewer/Reviewerinnen für ihre hilfreichen Kommentare.

Funding Open Access funding provided by Projekt DEAL.

Open Access Dieser Artikel wird unter der Creative Commons Namensnennung 4.0 International Lizenz veröffentlicht, welche die Nutzung, Vervielfältigung, Bearbeitung, Verbreitung und Wiedergabe in jeglichem Medium und Format erlaubt, sofern Sie den/die ursprünglichen Autor(en) und die Quelle ordnungsgemäß nennen, einen Link zur Creative Commons Lizenz beifügen und angeben, ob Änderungen vorgenommen wurden.

Die in diesem Artikel enthaltenen Bilder und sonstiges Drittmaterial unterliegen ebenfalls der genannten Creative Commons Lizenz, sofern sich aus der Abbildungslegende nichts anderes ergibt. Sofern das betreffende Material nicht unter der genannten Creative Commons Lizenz steht und die betreffende Handlung nicht nach gesetzlichen Vorschriften erlaubt ist, ist für die oben aufgeführten Weiterverwendungen des Materials die Einwilligung des jeweiligen Rechteinhabers einzuholen.

Weitere Details zur Lizenz entnehmen Sie bitte der Lizenzinformation auf http://creativecommons.org/licenses/by/4.0/deed.de.

\section{Literatur}

Amin A (2002) Ethnicity and the multicultural city: living with diversity. Report for the ESRC CITIES Programme and the Report for the Department of Transport, Local Government and the Regions. http://red.pucp.edu.pe/ridei/wp-content/uploads/ biblioteca/Amin_ethnicity.pdf. Zugegriffen: 1. März 2016

BBSR - Bundesinstitut für Bau-, Stadt- und Raumforschung (2015) Gemeinschaftsgärten im Quartier. BBSR-Online-Publikation 12, BBSR, Bonn

Bochynek-Friske A (2001) Ein Stadtteil von Hannover. Der Sahlkamp, Unser Zuhause. Selbstverlag, Hannover

Bourdieu P (1991) Physischer, sozialer und angeeigneter physischer Raum. In: Wentz M (Hrsg) Stadt-Räume. Campus, Frankfurt a. M., New York, S 25-34 
Carmona M, de Magalhães C, Hammond L (2008) Public space. The management dimension. Routledge, London, New York

Dangschat JS (2011) Partizipation, Integration und öffentlicher Raum. In: eNewsletter Netzwerk Bürgerbeteiligung 01/2011 vom 12.12.2011. Netzwerk Bürgerbeteiligung, Bonn

Gehl J (1987) Life between buildings. Using public space. Van Nostrand Reinhold, New York

ISG Hannover - Internationale StadtteilGärten Hannover e. V. (2017) Der gemeinnützige Verein „Internationale StadtteilGärten Hannover e. V.“. http://www.isghannover.de/ueber-uns/was-ist-interkul turelles-gaertern/. Zugegriffen: 22. Dezember 2019

Jacobs J (1993) Tod und Leben großer amerikanischer Städte, 3. Aufl. Vieweg, Braunschweig, Wiesbaden (Erstausgabe 1963)

Lapina L (2017) "Cultivating integration"? Migrant space-making in urban gardens. J Intercult Stud 38(6):621-636

LHH - Landeshauptstadt Hannover, Fachbereich Planen und Stadtentwicklung (2013) Freiraumentwicklungskonzept - Dokumentation. LHH, Hannover

LHH - Landeshauptstadt Hannover, Fachbereich Planen und Stadtentwicklung (2015) Integriertes Entwicklungskonzept 2015 Sahlkamp-Mitte. Sanierung „Soziale Stadt“, Hannover

LHH - Landeshauptstadt Hannover, Koordinierungsstelle Soziale Planung (2018) Sozialbericht 2018. Soziale Lagen, Stadtteile und Generationen, Hannover
Madanipour A (2010) Whose public space? In: Madanipour A (Hrsg) Whose public space? International case studies in urban design and development. Routledge, London, New York, S 237-243

Sennett R (1991) Civitas. Die Großstadt und die Kulturen des Unterschieds. S. Fischer, Frankfurt a. M.

Shaftoe H (2008) Convivial urban spaces. Creating effective public places. Earthscan, London

Valentine G (2008) Living with difference. Reflections on geographies of encounter. Prog Hum Geogr 32:323-337

Vertovec S (2007) Super-diversity and its implications. Ethn Racial Stud 30(6): 1024-1054

vhw - Verband Wohnen und Stadtentwicklung (Hrsg) (2017) Öffentliche Räume in stadtgesellschaftlich vielfältigen Quartieren: Nutzung, Wahrnehmung und Bedeutung. vhw, Aachen

Wiesemann L (2015) Öffentliche Räume und Diversität. Geographien der Begegnung in einem migrationsgeprägten Quartier - das Beispiel Köln-Mülheim. LIT, Berlin, Münster

Wood P, Landry C (2008) The intercultural city: planning for diversity advantage. Earthscan, London 